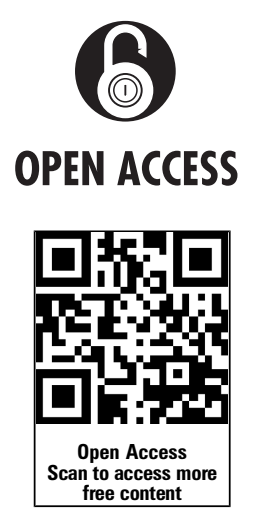

Handling editor Tore K Kvien

- Additional material is published online only. To view please visit the journal online (http://dx.doi.org/10.1136/ annrheumdis-2012-202485)

${ }^{1}$ Rheumatology Department, AP-HP, St-Antoine Hospital,

Paris, France

${ }^{2}$ Rheumatology Centre,

Paris, France

${ }^{3}$ Rheumatology Department,

AP-HP, Henri Mondor Hospital,

Créteil, France

${ }^{4}$ Department of Statistics,

DM Consultant, Angers, France

${ }^{5}$ Medical Department,

Laboratoires Expanscience,

Courbevoie, France

${ }^{6}$ Rheumatology B Department,

Paris-Descartes University;

AP-HP, Cochin Hospital,

Paris, France

${ }^{7}$ Purpan Hospital, Paul-

Sabatier-Toulouse 3, University and Rheumatology Centre,

Toulouse, France

${ }^{8}$ Twin Research, King's College

London, London, UK

${ }^{9}$ Department of Biostatistics,

Rouen University, Rouen,

France

${ }^{10}$ Rheumatology Department, Léopold Bellan Hospital,

Paris, France

Correspondence to

Dr E Maheu, Rheumatology Department, AP-HP, St-Antoine Hospital, 4, Bd Beaumarchais, Paris 75011, France; emaheu@wanadoo.fr

Received 10 August 2012 Revised 27 November 2012 Accepted 29 December 2012 Published Online First 23 January 2013

To cite: Maheu E, Cadet C, Marty $\mathrm{M}_{\text {, et al. Ann Rheum }}$ Dis 2014;73:376-384.

\title{
Randomised, controlled trial of avocado-soybean unsaponifiable (Piascledine) effect on structure modification in hip osteoarthritis: the ERADIAS study
}

\author{
Emmanuel Maheu, ${ }^{1}$ Christian Cadet, ${ }_{1}^{2}$ Marc Marty, ${ }^{3}$ Dominique Moyse, ${ }^{4}$ \\ Isabelle Kerloch ${ }^{5}$ Philippe Coste, ${ }^{5}$ Maxime Dougados, ${ }^{6}$ Bernard Mazières, ${ }^{7}$ \\ Tim D Spector, ${ }^{8}$ Hafid Halhol, ${ }^{5}$ Jean-Marie Grouin, ${ }^{9}$ Michel Lequesne ${ }^{10}$
}

ABSTRACT

Objective To assess the ability of avocado-soybean unsaponifiable-Expanscience (ASU-E) to slow radiographic progression in symptomatic hip osteoarthritis (OA).

Methods Prospective, randomised, double blind, parallel group, placebo controlled 3 year trial. Patients with symptomatic (painful $\geq 1$ year, Lequesne Index between 3 and 10) hip OA (American College of Rheumatology criteria) and a minimum joint space width (JSW) of the target hip between 1 and $4 \mathrm{~mm}$ on a pelvic radiograph were randomly assigned to $300 \mathrm{mg} /$ day ASU-E or placebo. Standing pelvis, target hip anteroposterior (AP) and oblique views were taken annually. The primary outcome was JSW change at year 3, measured at the narrowest point on pelvic or target hip AP view (manual measure using a $0.1 \mathrm{~mm}$ graduated magnifying glass). The full analysis dataset (FAS) included all patients having at least two successive radiographs. An analysis of covariance Mixed Model for Repeated Measurements with Missing at Random (for missing data) was performed to compare adjusted 3 year JSW changes (primary outcome) and the percentages of 'progressors' ( JSW loss $\geq 0.5 \mathrm{~mm}$ ) between groups.

Results 399 patients were randomised (345 kept in the FAS), aged 62 (35-84) years, 54\% women, mean body mass index 27 (SD 4) $\mathrm{kg} / \mathrm{m}^{2}$, mean symptom duration 4 (SD 5) years, 0-100 normalised Lequesne Index 30 (SD 9) and global pain visual analogue scale 37 (SD 23) $\mathrm{mm}$. Mean baseline JSW was $2.8(0.9) \mathrm{mm}$. There was no significant difference on mean JSW loss $(-0.638 \mathrm{~mm}$ vs $-0.672 \mathrm{~mm}, \mathrm{p}=0.72$, in the ASU-E and placebo groups, respectively) but there were $20 \%$ less progressors in the ASU-E than in the placebo group ( $40 \%$ vs $50 \%$, respectively, $p=0.040$ ). No difference was observed on clinical outcomes. Safety was excellent. Conclusions 3 year treatment with ASU-E reduces the percentage of JSW progressors, indicating a potential structure modifying effect in hip OA to be confirmed, and the clinical relevance requires further assessment.

Trial registration number on ClinicalTrial.gov NCT01062737

\section{INTRODUCTION}

Osteoarthritis (OA) is the most common joint disease. It weights a heavy burden. Hip OA affects about $10 \%$ of the population aged $65-73$ years. ${ }^{1}$ Its prevalence dramatically increases with age. ${ }^{2}$
Each year, 150000 total hip replacements (THR) are performed for OA in France and 100000 in the UK. It accounts for nearly $50 \%$ of the economic cost of OA in France or in Australia. ${ }^{3}{ }^{4}$ This burden is likely to dramatically increase ${ }^{5}$ : by 2030 , the demand for total hip arthroplasties for all conditions should grow by $174 \%$ in the USA and by $149 \%$ in The Netherlands. ${ }^{6} 7$

At present, there is no specific therapy targeting the pathological process of $\mathrm{OA}^{8}$ Several treatment options are available. ${ }^{9-11}$ Many symptom modifying therapies have been proposed with various levels of evidence. ${ }^{12}$ However, we still lack an approved disease modifying therapy because no treatment has proven without doubt its efficacy in preventing, stopping or delaying the disease, ${ }^{9-18}$ although glucosamine sulfate, chondroitin sulfate and very recently strontium ranelate have shown structure modifying properties in long term trials. ${ }^{19}$ Most of the OA structure modifications trials have been performed in knee OA, but three conducted in hip OA had unconvincing efficacy. ${ }^{20-22}$ The structural progression of $\mathrm{OA}$ is currently recommended to be assessed on plain radiographs by measuring joint space width (JSW) and joint space narrowing (JSN) over time, ${ }^{13-26}$ as clearly restated by the European Medicines Agency in 2010. ${ }^{26}$

Avocado-soybean unsaponifiable-Expanscience (ASU-E) (Laboratoires Expanscience, Courbevoie, France) is made up of unsaponifiable fractions of one-third avocado and two-third soybean oils (ie, extracts) and has shown anti-OA properties in preclinical in vitro and in vivo works. It has an inhibitory effect on interleukin 1 , a stimulating effect on collagen synthesis in articular chondrocyte cultures and a potential action on subchondral bone osteoblasts. ${ }^{27-36}$ ASU-E demonstrated efficacy on symptoms in hip and knee OA in some trials, ${ }^{37-39}$ which has been confirmed in a recent review and a meta-analysis. $^{40} 41$ A pilot 2 year placebo controlled structure modification trial on 163 patients did not show any significant structure modifying effect, but identified a significant reduction in JSN in the most radiologically severe patients subgroup (baseline JSW $<2.45 \mathrm{~mm}$ ), in a post-hoc analysis. ${ }^{21}$

We decided to perform a long term randomised controlled trial to assess the structure modifying effect of ASU-E in a large sample of symptomatic hip OA patients. 


\section{PATIENTS AND METHODS}

\section{Patients}

Outpatients, aged 45-75 years, were recruited by 122 French centres (52 private rheumatologists and 70 general practitioners) between February 2000 and January 2004.

\section{Definition of the disease}

Patients were suffering from idiopathic hip OA, fulfilling the American College of Rheumatology clinicoradiographic criteria. $^{42}$

\section{Symptom level at baseline}

Patients were symptomatic (constant or intermittent pain) for at least 1 year, with pain being present half of the time at least of the 3 months preceding selection, with a Lequesne AlgoFunctional Index score (range $0-24)^{43}$ for hip OA between 3 and 10, despite analgesics. In the case of bilateral complaints, the most symptomatic hip was chosen as the target hip.

\section{Radiographic severity at baseline}

Radiographic criteria for selection were a localised superolateral or superomedial JSN with a minimal JSW (narrowest site) between 1 and $4 \mathrm{~mm}$ on the target hip anteroposterior (AP) view, or in case of a concentric global JSN, a JSW between 1 and $4 \mathrm{~mm}$ on this view and reduced by at least $1 \mathrm{~mm}$ compared with the contralateral hip on the front pelvic view. These values account for $20 \%$ (JSW reduced by $1 \mathrm{~mm}$ ) and $80 \%$ (remaining joint space $\geq 1 \mathrm{~mm}$ ) of a normal hip joint space of $5 \mathrm{~mm}$ on average. ${ }^{44}$ Prior to randomisation, radiographic eligibility was centrally appraised by an independent observer (EM) who verified the above mentioned conditions and the quality of radiographs, according to predefined quality criteria (below). In addition, patients had to provide written informed consent to enter the study.

The main exclusion criteria were: secondary hip OA (as defined by Schumacher ${ }^{45}$ ) such as post-traumatic OA, congenital subluxation, acetabular dysplasia, avascular femoral head necrosis, inflammatory arthritis, metabolic arthritis, chondrocalcinosis (calcium deposition on the hip cartilage), Paget's disease or haemophilic arthritis; a high level of pain and few radiographic damages; posterior hip OA (posterior/inferoposterior JSN on Lequesne's oblique view ${ }^{46}$; patients likely to undergo a total joint replacement within the next 6 months; homolateral symptomatic knee OA; oral or parenteral corticosteroids during the previous month; intra-articular injection during the previous 3 months (except radiocontrast); and any serious concomitant medical illness.

\section{Study design}

This was a prospective, multicentre, randomised, double blind, placebo controlled, parallel group trial of 3 years' duration. The study protocol was approved by the ethics review board of the Pitié-Salpêtrière Hospital (Paris, France).

\section{Drug administration and concomitant therapies}

After confirmation that they fulfilled the selection criteria and written informed consent had been obtained, patients were randomly assigned to either the $300 \mathrm{mg}$ capsule of ASU-E group (Piascledine 300; Laboratoires Expanscience) or to the placebo capsule group, daily, for 3 years.

The randomisation list was previously established by an independent company (Creapharm, Le Haillan, France) by blocks of two for each stratum defined by baseline JSW: $<2.5 \mathrm{~mm}$ and $\geq 2.5 \mathrm{~mm}$ (according to a previous trial ${ }^{21}$ ). Treatment units were prepared by the same company in boxes of 3 months of treatment.

Treatment allocation was done following the central check of radiological selection criteria determining the stratum by the investigator following a numerical order. Treatment units were delivered by the investigator.

\section{Concomitant medications}

The use of analgesics and non steroidal anti-inflammatory drugs (NSAIDs) for OA symptoms was allowed after entry but had to be as low as possible and recorded. The amount taken was recorded by the patient using a self-report weekly diary. The investigator recorded the intake for each given period since the previous visit. Corticosteroid injections (periarticular around the target hip or intra-articular) were not allowed during the trial. Steroid injections in another articular site were allowed, if judged necessary by the investigator. Other symptom modifying drugs for OA were prohibited during the study period, as was indomethacin. ${ }^{47}$

\section{Treatment observance}

Treatment units given to the patient had to be returned at each visit to count and report study drug intake.

\section{Radiological and clinical outcomes}

The primary outcome was the change in JSW on the AP target hip view (or on the pelvic view if not available) ${ }^{48}$ at year 3 (or at the end of the study if not available). Three views were taken at selection and then yearly (12 views per patient): a pelvic front AP view (beam centred on the pubis), a target hip AP view and a target hip oblique view ('false profile ${ }^{\text {,6 }}$ ). AP target hip and pelvic views were performed according to a standardised protocol: (1) in the standing position ${ }^{49} 50$; (2) with standard distance from patient to radiography source of $1 \mathrm{~m}$; (3) with an internal feet rotation of $15^{\circ} \pm 5^{\circ}$ for front views; and (4) either plain or digitalised radiographs, provided they were made at the real size $(1 / 1$ or between $97 \%$ and $105 \%$ of the standard size if digitalised).

\section{Radiographic selection of patients}

The three views performed at selection were sent to the central reader who verified the patient's eligibility, and measured JSW to determine in which stratum the patient should be randomised. Then, the central reader sent his assessment to the investigator and the radiographs to the $\mathrm{CRO}$ which stored the $\mathrm{x}$-rays for the final reading of the primary outcome.

\section{Central radiographic measurement of joint space width}

Before unblinding the patient's data, it was planned to assess the performance (intra-/interobserver reliabilities and sensitivity to change) of the 'manual' chondrometry (as described by Lequesne $)^{50}$ and a computer assisted measurement. The methods and results of these assessments have been described elsewhere 4851 and, as a consequence, the 'manual' radiochondrometry with the best reader (CC) was therefore selected to perform the radiographic measurements. In addition, the performance of the three pelvic views was assessed. All baseline pelvic radiographs were also scored according to the KellgrenLawrence grading system. ${ }^{52}$

\section{Radiographic reading procedure}

Radiographs were anonymised for the time sequence by a random attribution of a letter for each visit at which radiographs 
Figure 1 Patient disposition in the trial (number of patients without any assessment of the primary outcome and number of protocol deviations were well balanced between the treatment groups). ASU-E, avocadosoybean unsaponifiable-

Expanscience.

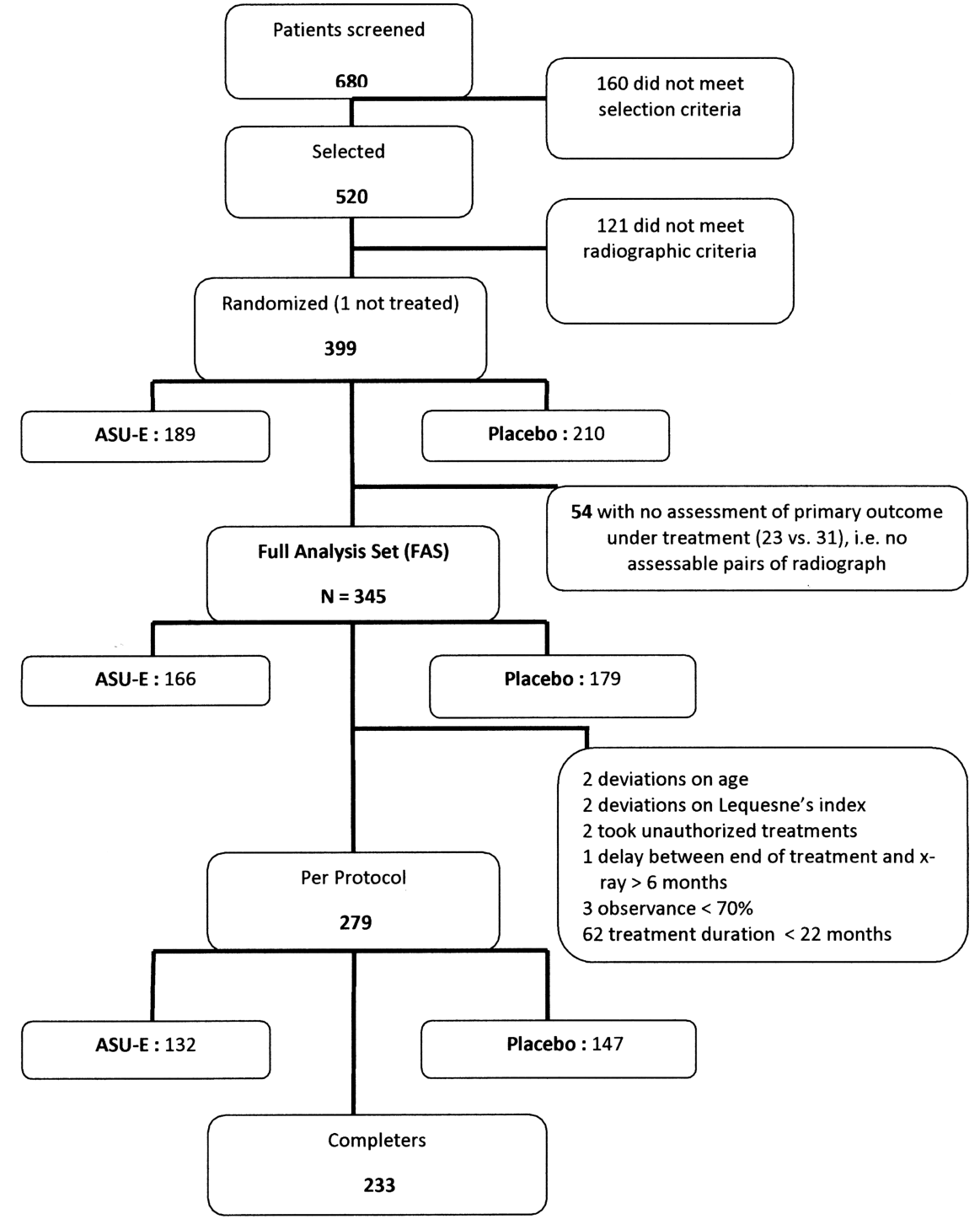

were performed. An envelope gathering all available views was labelled with the patient's trial number. The reader measured the minimal JSW on all radiographs of a given patient during the same session on a horizontal screen.

\section{Clinical assessments}

Clinical assessments for efficacy and safety parameters were recorded at months 1,3 and every 3 months until month 36, including: Lequesne's Index $(0-24)^{43}$ normalised on a $0-100$ basis; the Western and Ontario MacMaster University (WOMAC) visual analogue scale (VAS) ${ }^{53}$; global hip pain level during the past $48 \mathrm{~h}$ on a $0-100 \mathrm{~mm} \mathrm{VAS}^{54}$; global handicap rated by the patient on a $0-100 \mathrm{~mm}$ VAS and an 11 point numerical scale ${ }^{55}$; patient's global assessment of disease severity on a $0-100 \mathrm{~mm}$ VAS; percentages of patients having taken at least one NSAID and/or analgesic); and overall efficacy assessment by the patient and investigator. THR of the target hip occurring during the study was also assessed on all randomised patients having received the treatment. Safety was evaluated by the incidence of adverse events (AE) in each group, their description, rate of withdrawals for AEs and overall patient and physician assessments. Adherence was assessed by an open question and by counting the number of returned capsules.

\section{Statistical methods}

Sample size

Sample size determination was based on the primary endpoint, the change in JSW between baseline and year 3, using previous data. ${ }^{20}{ }^{21}$ Expecting a difference of $0.25 \mathrm{~mm}$ versus placebo in mean JSW change at year 3 and a common SD of $0.75 \mathrm{~mm}$,

\begin{tabular}{|c|c|c|c|}
\hline Patient status & Placebo (n (\%)) & ASU-E (n (\%)) & Total (n (\%)) \\
\hline Randomised patients & $210(100)$ & $189(100)$ & $399(100)$ \\
\hline Completers & $127(60.5)$ & $106(56.1)$ & $233(58.4)$ \\
\hline Premature withdrawals & $83(39.5)$ & $83(43.9)$ & $166(41.6)$ \\
\hline $\begin{array}{l}\text { Inefficacy with total } \\
\text { joint replacement }\end{array}$ & $36(17.1)$ & $38(20.1)$ & $74(18.5)$ \\
\hline $\begin{array}{l}\text { Inefficacy without total } \\
\text { joint replacement }\end{array}$ & $9(4.3)$ & $8(4.2)$ & $17(4.3)$ \\
\hline Adverse events & $11(5.2)$ & $10(5.3)$ & $21(5.3)$ \\
\hline Consent withdrawal & $11(5.2)$ & $17(9.0)$ & $28(7.0)$ \\
\hline Lost to follow-up & $8(3.8)$ & $3(1.6)$ & $11(2.8)$ \\
\hline Other & $7(3.3)$ & $6(3.2)$ & $13(3.3)$ \\
\hline Deaths & $1(0.5)$ & $1(0.5)$ & $2(0.5)$ \\
\hline
\end{tabular}


Table 2 Patient demographic and baseline characteristics in the full analysis set

\begin{tabular}{|c|c|c|c|}
\hline Characteristic & $\begin{array}{l}\text { Placebo } \\
(n=179)\end{array}$ & $\begin{array}{l}\text { ASU-E } \\
(n=166)\end{array}$ & $\begin{array}{l}\text { Total } \\
(n=345)\end{array}$ \\
\hline Gender (\% women) & 56 & 51 & 54 \\
\hline Age (years) (mean (SD)) & $62.7(8.0)$ & $61.6(7.9)$ & $62.2(7.9)$ \\
\hline BMI $\left(\mathrm{kg} / \mathrm{m}^{2}\right)$ (mean (SD)) & $26.8(4.4)$ & $27.0(4.1)$ & $26.9(4.2)$ \\
\hline $\begin{array}{l}\text { Delay since 1st symptoms (years) } \\
\text { (mean (SD)) }\end{array}$ & $4.3(4.6)$ & $4.4(5.6)$ & $4.3(5.1)$ \\
\hline $\begin{array}{l}\text { Delay since regular symptoms } \\
\text { (years) (mean (SD)) }\end{array}$ & $1.6(2.0)$ & $1.6(1.8)$ & $1.6(1.9)$ \\
\hline Other osteoarthritic sites (\% yes) & 56 & 50 & 53 \\
\hline Concomitant medications (\% yes) & 82 & 80 & 81 \\
\hline \multicolumn{4}{|l|}{ Kellgren-Lawrence grade (n (\%)) } \\
\hline I & $12(6.7)$ & $19(11.4)$ & $31(9)$ \\
\hline ॥ & $99(55.3)$ & $88(53.0)$ & $187(54.2)$ \\
\hline III & $66(36.9)$ & $59(35.5)$ & $125(36.2)$ \\
\hline IV & $2(1.1)$ & 0 & $2(0.6 \%)$ \\
\hline \multicolumn{4}{|l|}{ Location of JSN at narrowest site (n (\%)) } \\
\hline Superolateral & $99(55.3)$ & $80(48.2)$ & $179(51.9)$ \\
\hline Superomedial & $42(23.5)$ & $40(24.1)$ & $82(23.8)$ \\
\hline Superior global & $38(21.2)$ & $46(27.7)$ & $84(24.3)$ \\
\hline $\begin{array}{l}\text { JSW at narrowest site of target hip } \\
(\mathrm{mm})(\text { mean (SD)) }\end{array}$ & $2.76(0.9)$ & $2.85(0.9)$ & $2.81(0.9)$ \\
\hline $\begin{array}{l}\text { Lequesne Index (normalised 0-100) } \\
\text { (mean (SD)) }\end{array}$ & $30.4(10.0)$ & $30.0(8.4)$ & $30.2(9.3)$ \\
\hline $\begin{array}{l}\text { WOMAC pain score }(0-100 \mathrm{~mm}) \\
\text { (mean (SD)) }\end{array}$ & $32.7(20.6)$ & $31.6(18.6)$ & $32.2(19.7)$ \\
\hline $\begin{array}{l}\text { WOMAC stiffness score }(0-100 \mathrm{~mm}) \\
\text { (mean (SD)) }\end{array}$ & $36.2(25.3)$ & $35.4(23.5)$ & $35.8(24.4)$ \\
\hline $\begin{array}{l}\text { WOMAC function score }(0-100 \mathrm{~mm}) \\
\text { (mean (SD)) }\end{array}$ & $31.9(21.5)$ & $31.4(20.5)$ & $31.7(21.0)$ \\
\hline $\begin{array}{l}\text { Global hip pain (0-100 mm) } \\
\text { (mean (SD)) }\end{array}$ & $36.6(23.9)$ & $37.4(23.1)$ & $37.0(23.5)$ \\
\hline $\begin{array}{l}\text { Global handicap, numerical scale } \\
(0-10) \text { (mean (SD)) }\end{array}$ & $4.4(1.9)$ & $4.4(1.8)$ & $4.4(1.9)$ \\
\hline $\begin{array}{l}\text { Patient's global assessment } \\
(0-100 \mathrm{~mm})(\text { mean (SD)) }\end{array}$ & $40.3(27.9)$ & $40.4(25.3)$ & $40.3(26.6)$ \\
\hline
\end{tabular}

380 patients (190 per treatment group) were required to achieve $90 \%$ power considering a two sided nominal significance level of 0.05 . With this sample size, an analysis performed in the subgroup of severe patients whose size was expected to be half the targeted sample size (ie, 180 patients) could still achieve $75 \%$ power to show a difference of $0.35 \mathrm{~mm}$ versus placebo.

\section{Analysis sets}

Different sets were defined. (1) Safety set: all patients taking at least one treatment dose. (2) Randomised set based on the intention to treat principle. (3) Full analysis set (FAS) ${ }^{56}$ : all randomised patients taking at least one study treatment dose and with at least one baseline and one post baseline $\mathrm{x}$-ray to assess JSN on either the target hip AP or the pelvic view. (4) Completer set: all FAS patients with both baseline and month 36 x-rays. (5) Per protocol (PP) set: all FAS patients without any major deviation and with a treatment duration of at least $24 \pm 2$ months.

FAS was considered as the primary efficacy analysis set. Sensitivity analyses were performed in other sets (intention to treat and PP sets) to investigate the results of robustness. ${ }^{56}$

\section{Statistical analyses}

Descriptive statistics (mean, median, SD, quartiles for continuous and frequencies for categorical variables) were used to assess baseline characteristics in both groups. No statistical tests were performed between groups according to CPMP recommendations. ${ }^{57}$ Statistical hypotheses were tested by two sided tests at the 5\% nominal level of significance; $95 \% \mathrm{CI}$ were considered. All analyses were performed with SAS V.9.2 software.

\section{Primary efficacy analyses}

The primary efficacy endpoint was the change in JSW from baseline to year 3, which was assumed to be normally distributed. The primary model was a Mixed Model for Repeated Measurements (MMRM), assuming an unstructured correlation matrix and adjusting for baseline JSW, visit (year 1, 2 and 3), treatment, treatment by visit and baseline by visit interactions. Differences in adjusted JSW means between treatment groups with 95\% CI were estimated at year 3 using this mixed model. A sensitivity analysis using the Last Observation Carried Forward method for handling of missing data was also performed.

Several years after the study started, international consensus ${ }^{23-25}$ recommended not choosing JSN as the primary endpoint as the distribution of the latter was found to be particularly asymmetric and not easily tractable with usual statistical methods. Consequently, an amendment to the study protocol was issued before unblinding, defining a secondary efficacy analysis of the primary endpoint - that is, analysis of a binary endpoint (progression vs non-progression at 3 years) derived from the continuous JSN. A patient was defined as a progressor when JSW loss was $\geq 0.5 \mathrm{~mm}$ at 3 years. This $0.5 \mathrm{~mm}$ cut-off was selected before unblinding, based on the available literature at the time of finalisation of the statistical analysis plan, including a recent consensus recommending defining the cut-off based on the smallest detectable difference in JSN assessment of the reader. ${ }^{25}$ Also, another definition of a progressor was proposed: either a year $3 \mathrm{JSN} \geq 0.5 \mathrm{~mm}$ or a THR. Progressors rates were compared between treatment groups using a

Table 3 Results of analyses on the primary outcome: joint space width change measured on anteroposterior pelvis or target hip views using manual radiochondrometry, and rate of progressors, at 3 years, in the full analysis set, according to the MAR-MMRM analysis model

\begin{tabular}{llllll}
\hline & Placebo $(\boldsymbol{n}=179)$ & ASU-E $(\boldsymbol{n}=166)$ & $\mathrm{p}$ Value & OR/Diff & 95\% Cl \\
\hline Change in JSW at the narrowest point $(\mathrm{mm})$ (mean (SEM)) & $-0.672(0.066)$ & $-0.638(0.070)$ & $0.723^{*}$ & $0.034(0.096)$ & -0.156 to 0.224 \\
Progressors (progressor=JS loss $\geq 0.5 \mathrm{~mm}$ at 3 years) (\%) & 50.3 & 40.4 & $0.040+$ & 1.613 & 1.023 to 2.543 \\
\hline
\end{tabular}

${ }^{*}$ ANOVA treatment effect.

tCochran-Mantel-Haenzel test adjusted on stratum.

ASU-E, avocado-soybean unsaponifiable-Expanscience; JSW, joint space width; MAR-MMRM, Missing at Random-Mixed Model for Repeated Measurements; OR, odds ratio ASU-E versus placebo. 
Figure 2 Distribution of joint space narrowing in both groups of treatment. ASU-E, avocado-soybean unsaponifiable-Expanscience.

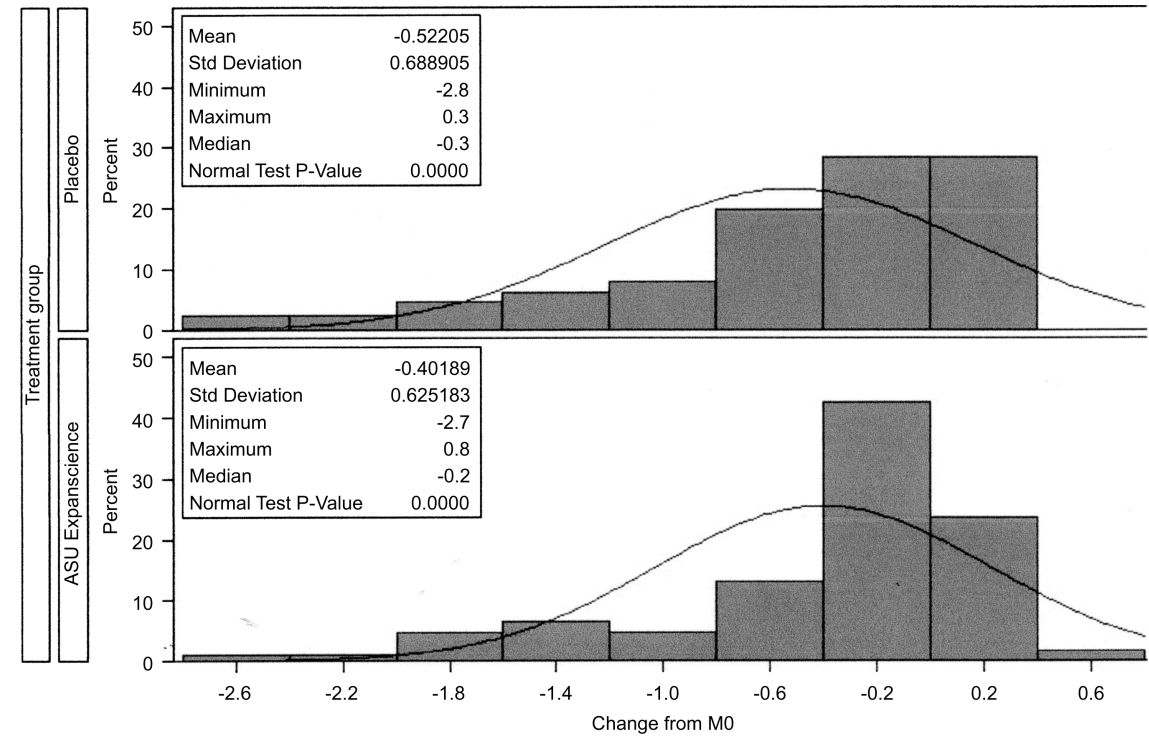

Cochran-Mantel-Haenszel test adjusted for severity stratum. A sensitivity logistic regression model adjusting for continuous baseline JSW was also fitted to compare the groups. ${ }^{56}$ Missing data were handled assuming Missing at Random (MAR). ${ }^{58}$ The MMRM which yields unbiased estimates of the treatment effect under this missing data mechanism was primarily favoured. ${ }^{58} 59$ The Last Observation Carried Forward method was also applied to check the sensitivity result to missing data. ${ }^{56}$ For the progressors analysis, year 3 missing values were predicted using both approaches.
Figure 3 Mean joint space narrowing (joint space width (JSW) change) and rates of progressors (JSN $\geq 0.5 \mathrm{~mm}$ vs baseline) at each time point, between baseline and year 3 in the full analysis set. ASU-E, avocado-soybean unsaponifiableExpanscience.

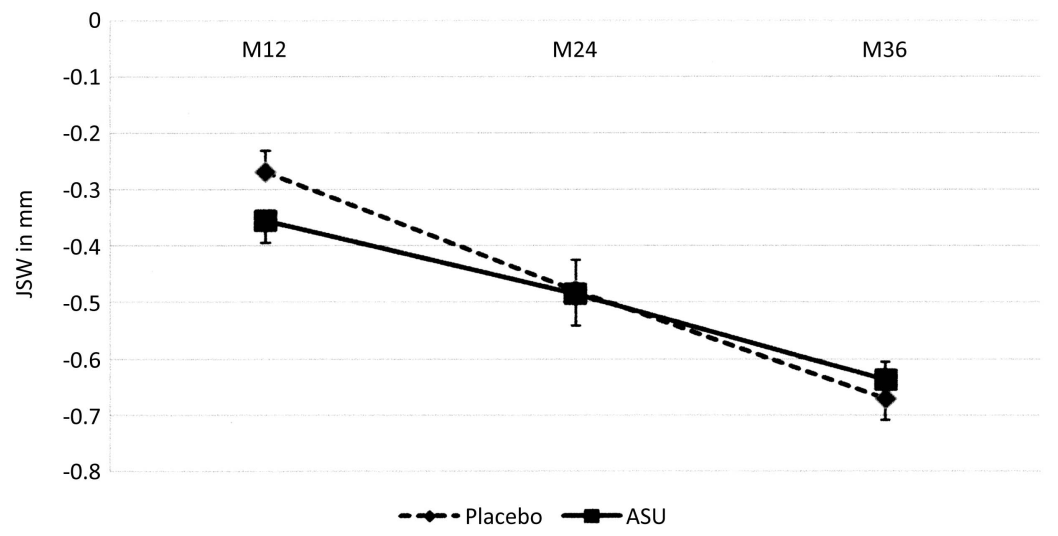

Joint Space Width change from baseline

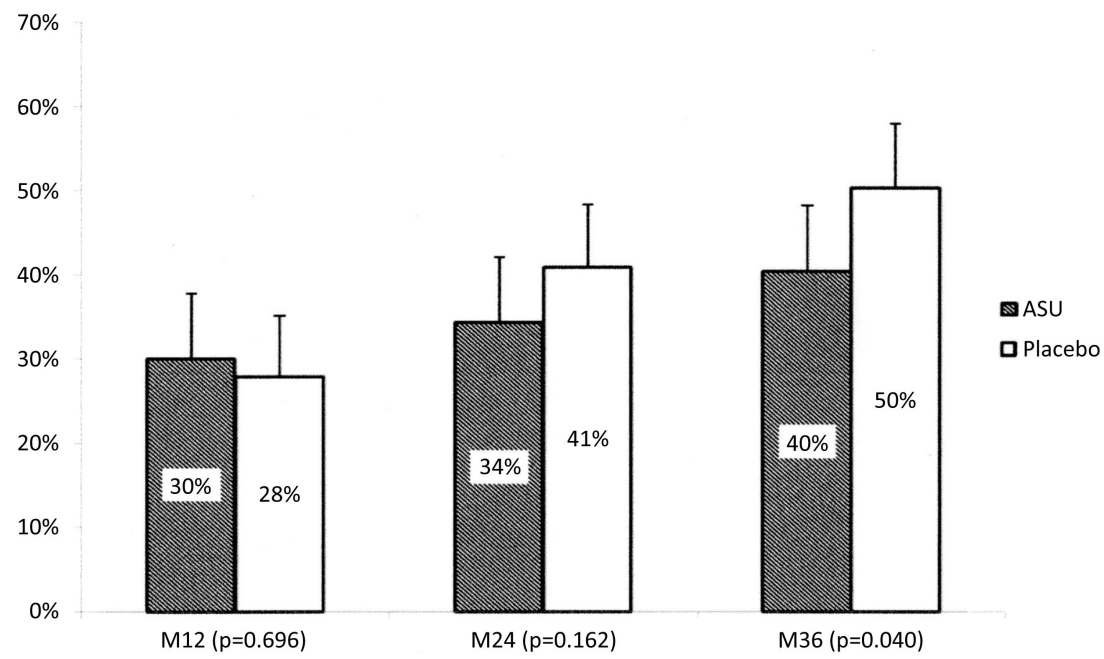

Rates of progressors in the two groups at each year (JSN $>0.5 \mathrm{~mm}$ vs baseline). 
Table 4 Clinical secondary outcomes between months 0 and 36 in the full analysis set (MAR-MMRM model)

\begin{tabular}{|c|c|c|c|c|c|}
\hline Clinical outcomes changes between months 0 and 36 & Placebo $(n=179)$ & ASU-E $(n=166)$ & p Value* & Difference & $95 \% \mathrm{Cl}$ \\
\hline Lequesne Index (normalised 0-100) & $-0.14(1.69)$ & $1.50(1.78)$ & 0.506 & $-1.63(2.45)$ & -6.45 to 3.19 \\
\hline WOMAC pain $(0-100 \mathrm{~mm})$ & $-0.98(2.17)$ & $-0.25(2.33)$ & 0.818 & $-0.73(3.18)$ & -7.00 to 5.54 \\
\hline WOMAC stiffness $(0-100 \mathrm{~mm})$ & $-2.29(2.23)$ & $-1.80(2.43)$ & 0.881 & $-0.49(3.30)$ & -6.99 to 6.01 \\
\hline WOMAC function $(0-100 \mathrm{~mm})$ & $2.10(2.13)$ & $1.48(2.30)$ & 0.843 & $0.62(3.13)$ & -5.55 to 6.79 \\
\hline Global hip pain on VAS (0-100 mm) & $-3.60(2.35)$ & $-4.26(2.51)$ & 0.849 & $0.66(3.43)$ & -6.10 to 7.42 \\
\hline Global handicap on $0-10$ scale & $-0.21(0.22)$ & $-0.53(0.24)$ & 0.326 & $0.32(0.32)$ & -0.32 to 0.96 \\
\hline Patient's global assessment $(0-100)$ & $-4.25(2.64)$ & $-4.42(2.84)$ & 0.963 & $0.18(3.87)$ & -7.45 to 7.81 \\
\hline
\end{tabular}

\section{Secondary efficacy outcome analyses}

Secondary clinical efficacy continuous endpoints were analysed at 6 months and at year 3 using the MMRM model. THR rates were compared using a Cochran-Mantel-Haenszel test stratified by severity and a Fisher exact test. Time to hip replacement (from baseline up to 6 months after trial cessation) was estimated by the Kaplan-Meier method and compared with the log rank test. Rates of patients taking NSAIDs and/or analgesics were compared using a $\chi^{2}$ test.

\section{Safety analyses}

AE were coded in the MedDRA dictionary (by organ class and preferred term). Comparison of $\mathrm{AE}$ rates between groups was performed by a Fisher exact test.

\section{RESULTS}

Disposition of patients in the study is described in figure 1. Of the 399 patients randomised, 189 belonged to the ASU-E and 210 to the placebo group; $345(86.5 \%)$ patients made up the FAS as there was no assessment of the primary outcome under treatment available for 54 patients. Reasons for premature withdrawals are listed in table 1 . The safety population included 398 patients as one randomised patient did not receive any study treatment while randomised.

Table 2 shows the demographic and baseline characteristics of the FAS.

\section{Primary endpoint}

On the FAS population using the MMRM missing replacement method, adjusted mean JSN at year 3 at the site of maximal narrowing was $-0.67 \mathrm{~mm}$ in the placebo group and $-0.64 \mathrm{~mm}$ in the ASU-E group, the difference of $0.034 \mathrm{~mm}(95 \% \mathrm{CI}-0.156$ to 0.225$)$ not being statistically significant $(p=0.72)$ (table 3$)$.

Table 5 Non-steroidal anti-inflammatory drug and analgesic intake between months 0 and 36 in the full analysis set

\begin{tabular}{llll}
\hline $\begin{array}{l}\text { Clinical outcome changes between } \\
\text { months } \mathbf{0} \text { and } 36\end{array}$ & $\begin{array}{l}\text { Placebo } \\
(\mathbf{n}=179)\end{array}$ & $\begin{array}{l}\text { ASU-E } \\
(\mathbf{n}=166)\end{array}$ & $\begin{array}{l}\mathbf{p} \\
\text { Value }\end{array}$ \\
\hline $\begin{array}{l}\text { NSAID/analgesic intake ( } \mathrm{n}(\%) \text { of } \\
\text { patients who had at least one intake of } \\
\text { an NSAID or analgesic during the trial) }\end{array}$ & $157(87.7)$ & $145(87.3)$ & 0.919 \\
$\begin{array}{l}\text { NSAID intake ( } \mathrm{n}(\%) \text { of patients who } \\
\text { had at least } 1 \text { intake during the trial) }\end{array}$ & $115(64.2)$ & $109(65.7)$ & 0.783 \\
\hline
\end{tabular}

ASU-E, avocado-soybean unsaponifiable-Expanscience; NSAID, non-steroidal anti-inflammatory drug.
As shown in figure 2, the distribution of JSN inside the groups was not statistically normal, but shifted to the right, indicating a high rate of patients with no JS deterioration and justifying the dichotomisation of JSN change into progressors/non-progressors.

The secondary efficacy analysis of the primary endpoint based on progressors at year 3 is given in table 3: 40.4\% of patients were classified as progressors in the ASU-E group versus $50.3 \%$ in the placebo group, with a statistically significant difference of approximately $10 \%(p=0.040)$. The relative risk reduction to be a progressor was $20 \%$ with ASU-E. Figure 3 indicates JSN and the rates of progressors at each time point.

The number needed to treat to obtain a non-progressor was 11 .

Indicative results of sensitivity analyses for both outcomes (JSN and progressors rates) are provided in an online supplementary file (S1). PP analyses yielded similar results: no statistical difference in JSN and a statistically significant difference between progressors rates favouring ASU-E, using the MAR-MMRM approach (see online supplementary file (S2)). The same results were observed with JSN measured by the computer assisted method (see online supplementary file (S3)).

Analysis of progressors rate using the secondary definition ( JSN $\geq 0.5 \mathrm{~mm}$ or THR) gave $51.4 \%$ of progressors in the placebo group versus $42.2 \%$ in ASU-E group $(p=0.054)$.

\section{Secondary endpoint analysis}

Regardless of the statistical method used to handle missing data, there was no between group difference on clinical outcomes: Lequesne Index, WOMAC (pain, stiffness, function), global pain, global handicap, patient's overall assessment (table 4) or analgesic/NSAID intake (table 5).

In the safety population, 83 patients $(20.9 \%)$ underwent a THR between months 0 and 42, with no between group difference (table 6). A higher percentage of THR was observed in the more severe patient stratum (JSW $<2.5 \mathrm{~mm}$ ): $37.5 \%$ vs $11.6 \%$, with no intergroup difference. Mean compliance with treatment was $97 \%$ in both groups.

\section{Safety analysis}

Mean exposure to treatment was 904 days in the placebo group and 880 in the ASU-E group $(p=0.348)$. Results of the safety analysis are presented in table 7 . Nearly $87 \%$ of patients reported at least one $\mathrm{AE}$ during the study and 32 patients reported at least one treatment related $\mathrm{AE}$; the most frequent AEs were musculoskeletal/connective tissue and infections/infestations. Sixty-three patients withdrew from the study for safety reason (no between group difference). 
Table 6 Number and percentages of patients who underwent a total hip replacement in the safety population $(n=398)$

\begin{tabular}{|c|c|c|c|c|}
\hline Patients who underwent THR & Placebo (209) & ASU-E (189) & Total (398) & p (Fisher) \\
\hline Between baseline and month $42(\mathrm{n}(\%))$ & $39 / 209 *(18.7)$ & $44 / 189 *(23.3)$ & $83 / 398(20.9)$ & 0.269 \\
\hline Baseline JSW<2.5 mm (n (\%)) & $27 / 77(35.1)$ & $27 / 67(40.3)$ & $54 / 144(37.5)$ & 0.605 \\
\hline Baseline JSW $\geq 2.5 \mathrm{~mm}(\mathrm{n}(\%))$ & $12 / 130(9.2)$ & $17 / 121(14.0)$ & 29/251 (11.6) & 0.244 \\
\hline Within 6 months after the end of the trial at patient level $(n(\%))$ & $35 / 209 *(16.7)$ & $38 / 189 *(20.1)$ & 73/398 (18.3) & 0.437 \\
\hline Baseline JSW<2.5 mm (n (\%)) & 23/77 (29.9) & $24 / 67(35.8)$ & $47 / 144(32.6)$ & 0.480 \\
\hline Baseline JSW $\geq 2.5 \mathrm{~mm}(\mathrm{n}(\%))$ & $12 / 130(9.2)$ & $14 / 121(11.6)$ & $26 / 251(10.4)$ & 0.679 \\
\hline
\end{tabular}

Safety was judged very good/good or moderate by $84.2 \%$ of patients in the placebo group and in $85.8 \%$ of patients in the ASU-E group.

\section{DISCUSSION}

Our main finding in this 3 year randomised controlled trial was that we observed no difference between the groups regarding JSW change, the continuous variable, at 3 years, but identified that ASU-E significantly decreased the rate of progressors to $40 \%$ compared with $50 \%$ in the placebo group, which represents a reduction by $20 \%$. Also, there was no between group difference in clinical parameters.

The main strength of our trial lies in the high level methodology used to evaluate the structural effect of ASU-E in hip OA. Indeed, it is now well known that assessing OA progression on radiographs by measuring JSW and JSN, although the current gold standard for Agencies and Scientific Societies, ${ }^{23-26}$ is problematic. For example: (1) reproducibility of the patient's positioning for taking radiographs; (2) reading method and selection of the reader(s); (3) definition of the primary outcome; (4) method of handling missing data, bearing in mind the $35-47 \%$ withdrawal rate in long term trials in symptomatic hip and knee OA usually reported, ${ }^{20} 4160-64$ in order to optimise the primary criterion analysis; and (5) definition of populations for the statistical analysis, particularly the intention to treat population. All of these issues were addressed as well as possibly using the highest standards of methodology and consensus working group discussions ${ }^{23-25}$ prior to breaking the code. As more scientific information arose during the trial, it became obvious that JSN in OA is not a quantitative linear normally distributed parameter. Indeed, many patients do not vary at all, even over a 3 year period (figure 2). We therefore planned a secondary major analysis of the primary endpoint taking into account this new knowledge while keeping our original primary outcome. A protocol amendment was instigated to analyse our primary outcome using the recommended binary approach: the progressors rates. ${ }^{25}$ This led to two analyses of the primary criterion, as reported. Only the latter analysis showed that ASU-E reduced the rate of progression in hip OA, compared with placebo. We defined our primary population (FAS) as patients having at least two measurements under treatment, on the same radiographic incidence, pelvis or target hip front view. Furthermore, we used the MMRM-MAR model, currently thought to be more accurate and clinically relevant for the purpose of trials than the Last Observation Carried Forward method using or not using the maximal bias, ${ }^{58} 59$ to estimate and analyse missing data. This allowed us to keep 112 premature dropouts in our primary analysis, giving 345 patients in the FAS (compared with 233 completers), and limited to 54 the number of patients lost for the primary analysis. This is the first 3 year structure modification trial in OA with such a low number of patients lost for the main analysis.

Our study has limitations. The first relates to the analysis of the primary criterion, since the analysis of the continuous

Table 7 Safety analyses in the ERADIAS trial

\begin{tabular}{|c|c|c|c|}
\hline No $(\%)$ of patients having: & $\begin{array}{l}\text { Placebo } \\
(n=209)\end{array}$ & $\begin{array}{l}\text { ASU-E } \\
(n=189)\end{array}$ & $\begin{array}{l}\text { Total } \\
(n=398)\end{array}$ \\
\hline At least one $A E(p=0.30)$ & $178(85.2)$ & $168(88.9)$ & $346(86.9)$ \\
\hline \multicolumn{4}{|l|}{ At least one $\mathrm{AE}$ of } \\
\hline 'Moderate to severe' intensity and unknown intensity & $160(76.6)$ & $146(77.3)$ & $306(76.9)$ \\
\hline 'Mild' intensity & $119(56.9)$ & $110(58.2)$ & $229(57.5)$ \\
\hline No of patients with at least one treatment related ${ }^{*} A E$ & $13(6.2)$ & $19(10.1)$ & $32(8.0)$ \\
\hline No of treatment related ${ }^{\star} \mathrm{AE}$ & $20(2.1)$ & $28(3.4)$ & $48(2.7)$ \\
\hline At least one $A E$ leading to treatment cessation & $31(14.8) \dagger$ & $32(16.9) \dagger$ & $63(15.8) \dagger$ \\
\hline At least one serious $A E(P=0.14)$ & $68(32.5) \ddagger$ & $75(39.7) \ddagger$ & $143(35.9) \ddagger$ \\
\hline At least one serious treatment related $\mathrm{AE}$ & $\begin{array}{l}2(1.0) \\
1 \text { hip replacement } \\
1 \text { wrist fracture }\end{array}$ & $\begin{array}{l}4(2.1) \\
3 \text { hip replacements } \\
1 \text { post surgery haematoma }\end{array}$ & $6(1.5)$ \\
\hline Deceased during the trial for treatment unrelated reasons & $\begin{array}{l}1 \text { glioblastoma } \\
1 \text { heart attack (ventricular } \\
\text { tachycardia) }\end{array}$ & $\begin{array}{l}1 \text { myocardial infarction with operated interventricular } \\
\text { communication }\end{array}$ & 3 \\
\hline
\end{tabular}


parameter (3 year JSN) did not show any between group difference, in contrast with the responder analysis which showed a significant relative reduction by $20 \%$ of progressors rate in the ASU-E group. A second point to consider is the absence of a symptomatic effect of ASU-E in this trial conversely to previous shorter symptom modification randomised controlled trials of ASU-E. There might be three reasons for this: (1) this trial was not designed for symptom modification but for structure modification; (2) enrolled patients had few symptoms at baseline in order to keep them in the trial and avoid too many premature dropouts for THR: $50 \%$ were at the patient acceptable symptom state ${ }^{65}$ for pain $(<35 \mathrm{~mm}$ on pain VAS) and/or function. Mean pain level was $37.0 \mathrm{~mm}$ and the mean normalised Lequesne Index score was 30.2 (7.2 in the 0-24 usual scoring); and (3) surprisingly, there was no placebo effect on pain or function, which is unusual in hip OA, as recently reviewed. ${ }^{66}$ It might be related to the fact that Zhang mostly identified the placebo effect in symptom modifying OA trials which included more symptomatic patients. However, it must be noticed that there was also no clinical effect observed in the structure modifying trials assessing diacerein ${ }^{20}$ or glucosamine ${ }^{22}$ in hip OA.

In this study, we did not evaluate the clinical relevance of the reduction in the numbers of progressors. A follow-up of patients is currently being performed with THR as the main outcome.

In summary, this is the first trial fulfilling the highest recommended methodological standards regarding the primary outcome measurement (JS loss) and its statistical analysis. It showed that 3 years of treatment with ASU-E did not reduce the average JS loss but reduced the percentage of JSW deteriorating patients compared with placebo, possibly indicating a potential structure modifying effect in hip OA. The clinical relevance of this requires further assessment.

Acknowledgements The authors are grateful to EURAXI Pharma Company, Joué les Tours, France, for monitoring of the centres and data management (Mr Sébastien Louveau). The authors are also grateful to Véronique Leblanc (Medical Director, Expanscience Labs) who carefully reviewed the manuscript and provided many useful comments. The authors thank all investigators, staff and patients for participation in this clinical trial.

Contributors EM designed the study, performed the radiographic prescreening and drafted the manuscript. CC contributed to the study design, data analysis and read all radiographs and performed the joint space measurements. MM contributed to the study design and gave statistical advice. DM performed the statistical analysis. $\mathrm{IK}, \mathrm{PC}$ and $\mathrm{HH}$ contributed to the study design. IK contributed to the data collection and storing. MD, BM, TDS, J-MG and ML contributed to the study design, data analysis and provided methodological advice. J-MG contributed highly to the statistical plan and analysis. ML was the main investigator of this trial. All authors had access to the data, critically reviewed and edited the manuscript, and approved the final version.

Funding This study was sponsored by Laboratoires Expanscience, Courbevoie, France.

Competing interests EM received consulting fees, support for travel to meetings and fees for board activities from Expanscience, consultancy fees from Pierre Fabre and Servier International, and received payment for lectures by Ibsa-Genevrier and Rottapharm. CC received consulting fees (for reading all the trial radiographs), support for travel to meetings and fees for board activities from Expanscience, consultancy fees from Servier International, and received payment for lectures by Expanscience and Rottapharm. MM received support for travel to meetings, fees for board activities from Expanscience and consultancy fees from Negma, and received payment for lectures by Pierre Fabre. DM received fees for board activities from Expanscience and performing the statistical analysis. IK, PC and $\mathrm{HH}$ are full time employees of Expanscience. MD received consulting fees and fees for board activities from Expanscience, and consultancy fees from Expanscience, Negma and Novartis. BM received consulting fees, support for travel to meetings and fees for board activities from Expanscience, consultancy fees from Pierre Fabre and Menarini, and payment for lectures by Pierre Fabre. TDS received consulting fees from Expanscience. J-MG received consulting fees and fees for board activities from Expanscience. ML received consulting fees, support for travel to meetings and fees for board activities, and for acting as the main investigator in this trial from Expanscience.
Ethics approval This trial was conducted in compliance with the Declaration of Helsinki and Good Clinical Practice Guidelines. The final protocol and all amendments to the protocol issued during the study and informed consent documentation were reviewed by the independent ethics committee of the Pitié-Salpêtrière Hospital, Paris, France.

Provenance and peer review Not commissioned; externally peer reviewed.

Open Access This is an Open Access article distributed in accordance with the Creative Commons Attribution Non Commercial (CC BY-NC 3.0) license, which permits others to distribute, remix, adapt, build upon this work non-commercially, and license their derivative works on different terms, provided the original work is properly cited and the use is non-commercial. See: http://creativecommons.org/ licenses/by-nc/3.0/

\section{REFERENCES}

1 Guillemin F, Rat AC, Mazieres B, et al. 3000 Osteoarthritis group. Prevalence of symptomatic hip and knee osteoarthritis: a two-phase population-based survey. Osteoarthritis Cartilage 2011;19:1314-22.

2 Pereira D, Peleteiro B, Araújo J, et al. The effect of osteoarthritis definition on prevalence and incidence estimates: a systematic review. Osteoarthritis Cartilage 2011;19:1270-85.

3 Le Pen C, Reygrobellet C, Gérentes I. Financial cost of osteoarthritis in France. The 'COART' France study. Joint Bone Spine 2005;72:567-70.

4 March LM, Bagga H. Epidemiology of osteoarthritis in Australia. Med J Australia 2004; 180:S6-10.

5 Ethgen 0, Reginster JY. Degenerative musculoskeletal disease. Ann Rheum Dis 2004:63:1-3.

6 Kurtz S, Onq K, Lau E, et al. Projections of primary and revision hip and knee arthroplasty in the United States from 2005 to 2030. J Bone Joint Surg Am 2007:89:780-5

7 Otten R, Van Roermund PM, Picavet HS. Trend in the number of knee and hip arthroplasties: considerably more knee and hip prostheses due to osteoarthritis in 2030. Ned Tijdschr Geneeskd 2010;154:A1534.

8 Berenbaum F. Targeted therapies in osteoarthritis: a systematic review of the trials on http://www.clinicaltrials.gov. Best Pract Res Clin Rheumatol 2010;24:107-19.

9 Jordan KM, Arden NK, Doherty M, et al. EULAR recommendations 2003: an evidence based approach to the management of knee osteoarthritis: report of a Task Force of the Standing Committee for International Clinical Studies Including Therapeutic Trials (ESCISIT). Ann Rheum Dis 2003;62:1145-55.

10 Zhang W, Doherty $M$, Arden $N$, et al. EULAR evidence based recommendations for the management of hip osteoarthritis: report of a task force of the EULAR Standing Committee for International Clinical Studies Including Therapeutics (ESCISIT). Ann Rheum Dis 2005;64:669-81.

11 Zhang W, Moskowitz RW, Nuki G, et al. OARSI recommendations for the management of hip and knee osteoarthritis, Part II: OARSI evidence-based, expert consensus guidelines. Osteoarthritis Cartilage 2008;16:137-62.

12 Zhang W, Nuki G, Moskowitz RW, et al. OARSI recommendations for the management of hip and knee osteoarthritis: part III: changes in evidence following systematic cumulative update of research published through January 2009. Osteoarthritis Cartilage 2010;18:476-99.

13 Lequesne $M$, Brandt K, Bellamy N, et al. Guidelines for testing slow acting drugs in osteoarthritis. J Rheumatol 1994;21(Suppl 41):65-71.

14 Bouvenot $\mathrm{G}$. Considerations on the methodology of studies evaluating treatments for osteoarthritis. Rev Rhum Engl Ed 1994;61:74-6.

15 Dougados M. Clinical assessment of osteoarthritis in clinical trials. Curr Opin Rheumatol 1995;7:87-91.

16 Altman $\mathrm{R}$, Brandt $\mathrm{K}$, Hochberg $\mathrm{M}$, et al. Design and conduct of clinical trials in patients with osteoarthritis: recommendations from a task force of the Osteoarthritis Research Society. Results of a Workshop. Osteoarthritis Cartilage 1996;4:217-43.

17 Dougados $M$, Devogelaer JP, Annefeld $M$, et al. (the Members of the Group for the Respect of Ethics and Excellence in Science). Recommendations for the registration of drugs used in the treatment of osteoarthritis. Ann Rheum Dis 1996:55:552-7.

18 Bellamy N, Kirwan J, Altman R, et al. Recommendations for a core set of outcome measures for future phase III clinical trials in knee, hip and hand osteoarthritis: consensus development at OMERACT III. J Rheumatol 1997;24:799-802.

19 Reginster JY, Badurski J, Bellamy N, et al. Efficacy and safety of strontium ranelate in the treatment of knee osteoarthritis: results of a double-blind, randomised placebo-controlled trial. Ann Rheum Dis 2013;72:179-86.

20 Dougados M, Nguyen M, Berdah L, et al. The ECHODIAH investigators study group. Evaluation of the structure-modifying effects of diacerein in hip osteoarthritis: ECHODIAH, a three-year, placebo-controlled trial. Evaluation of the chondromodulating effect of diacerein in OA of the hip. Arthritis Rheum 2001:44:2539-47.

21 Lequesne M, Maheu E, Cadet C, et al. Structural effect of avocado/soybean unsaponifiables on joint space loss in osteoartritis of the hip. Arthritis Rheum Arthritis Rheum 2002;47:50-8. 
22 Rozendaal RM, Koes BW, van Osch GJ, et al. Effect of glucosamine sulfate on hip osteoarthritis: a randomized trial. Ann Intern Med 2008;148:268-77.

23 Altman RD, Bloch DA, Dougados $\mathrm{M}$, et al. Measurement of structural progression in osteoarthritis of the hip: the Barcelona consensus group. Osteoarthritis Cartilage 2004; 12:515-24.

24 Abadie E, Ethgen D, Avouac B, et al. Recommendations for the use of new methods to assess the efficacy of disease-modifying drugs in the treatment of osteoarthritis. Osteoarthritis Cartilage 2004;12:263-8.

25 Ornetti P, Brandt K, Hellio Le Graverand MP, et al. OARSI-OMERACT definition of relevant radiological progression in hip/knee osteoarthritis. Osteoarthritis Cartilage 2009;17:856-63.

26 EMEA-CHMP. Guideline on clinical investigation of medicinal products used in the treatment of osteoarthritis. CPMP/EWP/784/97 Rev. 1. http://www.ema.europa.eu/ ema/index.jsp?curl.../document.../document...search ... (accessed 20 Jan 2010).

27 Mauviel A, Daireaux M, Hartmann DJ, et al. Effets des insaponifiables d'avocat et de soja (PIAS) sur la production de collagène par des cultures de synoviocytes, chondrocytes articulaires et fibroblastes dermiques. Rev Rhum Mal Ostéoartic 1989;56:207-11.

28 Mauviel A, Loyau G, Pujol JP. Effet des insaponifiables d'avocat/soja (Piasclédine ${ }^{\circledR}$ ) sur l'activité collagénolytique de cultures de synoviocytes rhumatoïdes humains et de chondrocytes articulaires de lapin traités par l'interleukine 1. Rev Rhum Mal Ostéoartic 1991;58:241-5.

29 Henrotin Y, Labasse A, Zheng SX, et al. Effects of three avocado/soybean unsaponifiable mixtures on human articular chondrocytes metabolism. Clin Rheumatol 1998;17:31-9.

30 Boumediene K, Felisaz N, Bogdanowicz P, et al. Avocado/soya unsaponifiables enhance expression of transforming growth factor- 1 and -2 in cultured articular chondrocytes. Arthritis Rheum 1999;42:148-56.

31 Henrotin YE, Sanchez C, Deberg MA, et al. Avocado/soybean unsaponifiables increase aggrecan synthesis and reduce catabolic and proinflammatory mediator production by human osteoarthritic chondrocytes. J Rheumatol 2003:30:1825-34.

32 Henrotin YE, Deberg MA, Crielaard JM, et al. Avocado/soybean unsaponifiables prevent the inhibitory effect of osteoarthritic subchondral osteoblasts on aggrecan and type II collagen synthesis by chondrocytes. J Rheumatol 2006;33:1668-78.

33 Gabay 0, Gosset M, Levy A, et al. Stress-induced signaling pathways in hyaline chondrocytes: inhibition by avocado-soybean unsaponifiables (ASU). Osteoarthritis Cartilage 2008;16:373-84.

34 Mazières $B$, Tempesta $C$, Tiechard $M$, et al. Pathologic and biochemical effects of a lipidic avocado and soya extract on an experimental post-contusive model of OA. Osteoarthritis Cartilage 1993:1:46.

35 Cake MA, Read RA, Guillou B, et al. Modification of articular cartilage and subchondral bone pathology in an ovine meniscectomy model of osteoarthritis by avocado and soya unsaponifiables (ASU). Osteoarthritis Cartilage 2000;8:404-11.

36 Boileau C, Martel-Pelletier J, Caron J, et al. Protective effects of total fraction of avocado/soybean unsaponifiables on the structural changes in experimental dog osteoarthritis: inhibition of nitric oxide synthase and matrix metalloproteinase-13. Arthritis Res Ther 2009;11:R41.

37 Blotman F, Maheu E, Wulwik A, et al. Midterm efficacy and safety of avocado and soya unsaponifiables (ASU) in the treatment of knee and hip osteoarthritis: results of a three-month prospective, randomized, double-blind, placebo-controlled, parallel groups, multicenter clinical trial. Rev Rhum Engl Ed 1997:64:825-34.

38 Maheu E, Mazières B, Valat JP, et al. Symptomatic efficacy of avocado/soybean unsaponifiables in the treatment of osteoarthritis of the knee and hip: a prospective, randomized, double-blind, placebo-controlled, multicenter clinical trial with a six-month treatment period and a two-month followup demonstrating a persistent effect. Arthritis Rheum 1998;41:81-91.

39 Appelboom T, Schuermans J, Verbruggen $G$, et al. Symptoms modifying effect of avocado/soybean unsaponifiables (ASU) in knee osteoarthritis. A double blind, prospective, placebo controlled study. Scand J Rheumatol 2001;30:242-7.

40 Ernst E. Avocado-soybean unsaponifiables (ASU) for osteoarthritis - a systematic review. Clin Rheumatol 2003;22:285-8.

41 Christensen R, Bartels EM, Astrup A, et al. Symptomatic efficacy of avocadosoybean unsaponifiables (ASU) in osteoarthritis (OA) patients: a meta-analysis of randomized controlled trials. Osteoarthritis Cartilage 2008:16:399-408.

42 Altman RD, Alarcon G, Appelrouth D, et al. Development of criteria for the classification and reporting of osteoarthritis. Classification of osteoarthritis of the hip. Arthritis Rheum 1991;34:505-14.
43 Lequesne $M$, Méry C, Samson $M$, et al. Indexes of severity for osteoarthritis of the hip and knee. Scand J Rheumatol 1987;65(Suppl):85-9.

44 Reis P, Nahal-Saïd R, Ravaud Ph, et al. Are radiological joint space widths of normal hips asymmetrical? Ann Rheum Dis 1999;58:246-9.

45 Schumacher RH, Chen LX, Buckwalter J. Secondary osteoarthritis. In: Moskowitz R, Altman RD, Hochberg M, et al. eds. Osteoarthritis. Philadelphia: Lippincott, 2007: 233-53.

46 Lequesne GM, Laredo JD. The faux profil (oblique view) of the hip in the standing position. Contribution to the evaluation of osteoarthritis of the adult hip. Ann Rheum Dis 1998:57:676-81.

47 Rashad S, Revell P, Hemingway A, et al. Effect of non-steroidal anti-inflammatory drugs on the course of osteoarthritis. Lancet 1989:2:519-22.

48 Maheu E, Cadet C, Marty M, et al. Reproducibility and sensitivity to change of various methods to measure joint space width in osteoarthritis of the hip: a double reading of three different radiographic views taken with a three-year interval. Arthritis Res Ther 2005;7:R1375-85.

49 Conrozier T, Lequesne MG, Tron AM, et al. The effects of position on the radiographic joint space in osteoarthritis of the hip. Osteoarthritis Cartilage 1997:5:17-22.

50 Lequesne M. Quantitative measurement of joint space during progression of osteoarthritis: "Chondrometry." In: Kuettner K, Goldberg V, eds. Osteoarthritis disorders. Rosemont, IL: American Academy of Orthopaedic Surgeons, 1995: 427-44.

51 Conrozier T, Brandt K, Piperno $\mathrm{M}$, et al. Reproducibility and sensitivity to change of a new method of computer measurement of joint space width in hip osteoarthritis. Performance of three radiographic views obtained at a 3-year interval. Osteoarthritis Cartilage 2009;17:864-70.

52 Kellgren JH, Lawrence DM. Radiological assessment of osteoarthritis. Ann Rheum Dis 1957;16:494-502.

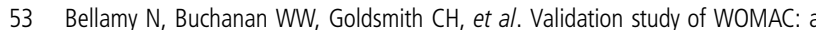
health status instrument for measuring clinically important patient relevant outcomes to antirheumatic drug therapy in patients with osteoarthritis of the hip or knee. J Rheumatol 1988;15:1833-40.

54 Huskisson EC. Measurement of pain. Lancet 1974:2:1127-31.

55 Lequesne MG, Maheu E. Clinical and radiological evaluation of hip, knee and hand osteoarthritis. Aging Clin Exp Res 2003;15:380-90.

56 Europ Agency Eval Med Products. International Conference Harmonisation (ICH) Topic E9-Step 4 Consensus Guideline: note for guidance on statistical principles for clinical trials. London: European Agency, 1998: 24-5.

57 EMEA-CHMP. CPMP/EWP/2863/99: Points to Consider on Adjustment for Baseline Covariates. 22 May 2003. http://www.ema.europa.eu/docs/eu_GB/ document_library/Scientific_guideline/2009/09/WC 500003639.pdf

58 Siddiqui 0 , Hung HM, O'Neill R. MMRM vs LOCF: a comprehensive comparison based on simulation study and 25 NDA datasets. J Biopharm Stat 2009;19:227-46.

59 Molenberghs G, Kenward MG. Missing data in clinical studies. New York: John Wiley \& Sons Ed, 2007.

60 Reginster JY, Deroisy R, Rovati LC, et al. Long term effects of glucosamine sulfate on osteoarthritis progression : a randomised, placebo-controlled clinical trial. Lancet 2001;357:251-6.

61 Pavelka K, Gatterová J, Olejarová M, et al. Glucosamine sulfate use and delay of progression of knee osteoarthritis. A 3-year, randomized, placebo-controlled, double-blind study. Arch Intern Med 2002;162:2113-23.

62 Spector TD, Conaghan PG, Buckland-Wright JC, et al. Effect of risedronate on joint structure and symptoms of knee osteoarthritis: results of the BRISK randomized, controlled trial. Arthritis Res Ther 2005;7:R625-33.

63 Brandt KD, Mazzuca SA, Katz BP, et al. Effect of doxycycline on progression of osteoarthritis. Results of a randomized, placebo-controlled, double-blind trial. Arthritis Rheum 2005;52:2015-25.

64 Kahan A, Uebelhardt D, De Vathaire F, et al. Long-term effects of chondroitin4 and 6 sulfate on knee osteoarthritis. The study on osteoarthritis progression prevention: a two-year randomized, double-blind, placebo-controlled trial. Arthritis Rheum 2009;60:524-33

65 Tubach F, Ravaud P, Baron G, et al. Evaluation of clinically relevant states in patient reported outcomes in knee and hip osteoarthritis: the patient acceptable symptom state. Ann Rheum Dis 2005;64:34-7.

66 Zhang W, Robertson J, Jones AC, et al. The placebo effect and its determinants in osteoarthritis: meta-analysis of randomised controlled trials. Ann Rheum Dis 2008;67:1716-23. 